\title{
The emergence of charge coherence in soft molecular organic semiconductors via the suppression of thermal fluctuations
}

\author{
Kenichi Sakai ${ }^{1}$, Yugo Okada ${ }^{1}$, Takafumi Uemura ${ }^{1}$, Junto Tsurumi ${ }^{1}$, Roger Häusermann ${ }^{1}$, Hiroyuki Matsui ${ }^{1}$, \\ Tatsuya Fukami ${ }^{2}$, Hiroyuki Ishii ${ }^{2}$, Nobuhiko Kobayashi ${ }^{2}$, Kenji Hirose ${ }^{2}$ and Jun Takeya ${ }^{1}$ \\ Organic semiconductors are already widely used in electronics. Nevertheless, their fundamental properties are still being \\ debated. In particular, charge transport, which determines the performance of organic light-emitting diodes, solar cells and \\ organic field-effect transistors, has been described by a wide range of complementary but incompatible theories. These theories \\ involve either localized charge carriers hopping from molecule to molecule, leading to incoherent charge transport, or delocalized \\ charge carriers moving around freely in the semiconductor, leading to coherent transport. In this communication, we reveal \\ the first experimental evidence that charge coherence can be tuned from partially to fully coherent in one and the same material \\ - pentacene-showing a continuous transition from one transport mechanism to the other. Microscopically, the transport \\ mechanism depends on the overlap of adjacent molecular orbitals, which in turn is sensitive to molecular thermal fluctuations. \\ We control these fluctuations through moderate variations of pressure and temperature, leading to a mobility increase of $75 \%$. \\ We quantify the influence of these thermal fluctuations by estimating the critical value below which fully coherent charge \\ transport emerges. The ability to control thermal fluctuations and therefore to effectively tune the charge coherence is an \\ important key to improving charge transport in soft molecular materials.
}

NPG Asia Materials (2016) 8, e252; doi:10.1038/am.2016.30; published online 25 March 2016

\section{INTRODUCTION}

Historically, the existence of coherent electronic states in weakly van der Waals bonded crystals has been a controversial topic. ${ }^{1}$ The weak intermolecular interaction leads to a strong thermal vibration of each molecule, resulting in the dynamic disorder of the crystal lattice. Dynamic disorder subsequently leads to a complex interaction between the soft lattice and the charge carriers, which is unique to these systems. In classical semiconductors, the charge transport mechanism can be directly determined from the temperature dependence of the mobility. In an organic semiconductor, however, the temperature dependence of the mobility does not necessarily reveal the nature of the charge transport and therefore it cannot be determined whether a system is described by the short-range hopping of localized charge carriers or band transport due to extended electronic wavefunctions. $^{2}$ For this reason, a more direct method is required to study the nature of charge transport in these systems. The Hall effect is such a method, as it is directly connected to the coherence of the charge carrier wavefunction via interaction with the applied magnetic field. The quantum mechanical origin of this interaction lies in the coupling of the wave number $\mathrm{k}$ of the delocalized charge carrier wavefunction with the vector potential A of the magnetic field.
This coupling is only possible if a wave number $\mathrm{k}$ can be assigned to the charge carrier. Therefore, the wavefunction must be coherent over several molecules. ${ }^{3-5}$ For these reasons, the experimental observation of the Hall effect in the hopping systems of localized charge carriers significantly differs from that of band-transport systems. More precisely, the inverse Hall coefficient $1 / R_{H}$ equals the charge density $Q$ only if the charge carriers are coherent. Indeed, incoherent electronic systems, such as amorphous silicon films, show very low response to an applied magnetic field. ${ }^{6}$ A similar observation has been reported for organic polymers: if they are dominated by static disorder, then they only show a weak Hall signal, ${ }^{7}$ whereas, in more ordered polymers, a free-electron-like wavefunction has been measured. ${ }^{8}$

In previous studies, we successfully measured the Hall effect in organic semiconductors using rubrene single-crystal transistors, 3,5 which are well known to be the highest performing organic transistors at room temperature, with a mobility above $20 \mathrm{~cm}^{2} \mathrm{Vs}^{-1}$. $^{9-11}$ The experiment showed precise measurements for the band-transport relation, that is, $R_{H}=1 / Q$, indicating coherent electronic states. Subsequently, materials as dinaphtho[2,3-b:2',3'-f] thieno[3,2-b] thiophene (DNTT), alkylated DNTT, pentacene derivatives and

${ }^{1}$ Graduate School of Frontier Sciences, The University of Tokyo, Chiba, Japan and ${ }^{2}$ Institute of Applied Physics, Tsukuba Research Center for Interdisciplinary Materials Science, University of Tsukuba, Ibaraki, Japan

Correspondence: Professor J Takeya, Graduate School of Frontier Sciences, The University of Tokyo, Takeya lab., 4F Kiban Building, 5-1-5 Kashiwanoha, Kashiwa, Chiba 277-8561, Japan.

E-mail: takeya@k.u-tokyo.ac.jp

Received 27 May 2015; revised 18 October 2015; accepted 24 November 2015 
N,N'-1H,1H-perfluorobutyldicyanoperylene-carboxydi-imide (PDIF$\mathrm{CN}_{2}$ ) turned out to satisfy the same condition, and coherent band transport was observed at room temperature. ${ }^{12-15}$ We then focused on pentacene field-effect transistors (FET), in which we found that only half of the Hall voltage appeared at room temperature, indicating an incomplete coherence. At lower temperatures, we found the coherence increased; however, even at $150 \mathrm{~K}$, charge transport was only partially coherent. ${ }^{16}$

Here, we report the emergence of fully coherent charge transport in pentacene single crystals under pressure. The measurements are performed by varying both temperature and pressure using the same experimental setup as in our previous studies. ${ }^{17-18}$ Both the field-effect properties and the Hall effect are measured on the same sample.

\section{MATERIALS AND METHODS}

\section{Growth of single crystals}

The pentacene single crystals were grown using the physical vapor transport method. ${ }^{2}$ After purifying crude powder samples, supplied by Sigma-Aldrich Japan K.K., Tokyo, Japan, by vaporizing at $277^{\circ} \mathrm{C}$ and coarse-crystallizing at $215^{\circ} \mathrm{C}$ in a two-zone tube furnace under Ar gas flow, the coarse crystals were slowly recrystallized at the same temperature within approximately 3 days.

\section{FET fabrication}

The FET samples, with their bottom-contact/bottom-gate structure, were fabricated as follows: a patterned gate electrode of $20 \mathrm{~nm}$ in thickness was vapor-deposited through a shadow mask on a polyethylene naphthalate substrate. A gate-insulating layer of Cytop (Asahi Glass Co., Tokyo, Japan) was formed by spin-coating and subsequent annealing at $140{ }^{\circ} \mathrm{C}$ for $15 \mathrm{~h}$. The thickness of the Cytop layer of $600 \mathrm{~nm}$ was estimated using atomic force microscopy. A single-crystal pentacene flake with a thickness of less than $1 \mu \mathrm{m}$ was laminated on top. The source-drain electrodes and four voltage terminals with a thickness of $20 \mathrm{~nm}$ were constructed by vapor deposition of gold layers through a shadow mask. Finally, the Hall-bar-shaped channel was produced using a laser etching set up so that the central channel was structured on the gate electrode (Figure 1). This structure ensures a nearly homogeneous shrinking of the whole device under elevated pressure. The width $\mathrm{W}$ and length $\mathrm{L}$ of the channels were 100 and $150 \mu \mathrm{m}$, respectively. The distance 1 between the adjacent voltage terminals along the channel direction was $50 \mu \mathrm{m}$. The device was covered with a few micrometer-thick parylene for passivation and then completely filled in epoxy (STYCAST 1266, Henkel Ablestik Japan Ltd., Yubari, Japan) for mechanical stability. The whole piece was introduced in a standard Cu-Be pressure cell. Fluorinert FC70/77 (3M Japan Ltd., Tokyo, Japan) was used as the pressurizing medium. ${ }^{17}$

\section{Gate capacitance $\mathrm{C}$ as a function of pressure}

Because the gate capacitance $C$ varies with the applied pressure, we can use the value of $C$ as a function of pressure, as identified in our previous study; we estimated the $V_{G}$ dependent carrier density at each pressure by Hall effect

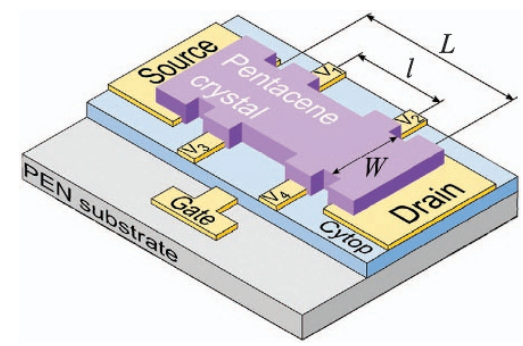

Figure 1 Device schematics for the gated Hall measurement as well as four-terminal FET setup. First, the pentacene single crystal was placed on pre-evaporated gold electrodes, and then a Hall-bar structure was cut using laser etching. The Hall voltage and field-effect performance were measured on the same sample. measurements using rubrene single-crystal FETs, where $C(P)$ was observed to change by $10 \%$ at $0.5 \mathrm{GPa}^{17}$

\section{Measurements of field-effect properties}

We use the four-terminal technique to analyze the FET characteristics to exclude the pressure dependence of the contact resistance. Our measurements of the field-effect properties show that the produced device exhibits the characteristics of a FET (Supplementary Figure S1).

As illustrated in Figure 1 , the voltages $V_{1}, V_{2}$ and $V_{3}$ are measured at different positions. The four-terminal sheet conductivity $\sigma$ and the Hall voltage $V_{H}$ are given by

$$
\sigma=\frac{W \quad I_{D}}{l V_{2}-V_{1}}, V_{H}=V_{3}-V_{1}
$$

where $\sigma$ is connected with the carrier mobility $\mu$ as follows:

$$
\mu=\frac{1 \delta \sigma}{C_{i} \delta V_{G}} .
$$

$I_{D}$ is the drain current, $V_{G}$ is the applied gate voltage and $C_{i}$ is the capacitance of the Cytop gate insulator per unit area. Figure 2a shows $\sigma$ plotted as a function of $V_{G}$ for various applied pressures. Increasing the pressure reduces the distance between the molecules. Therefore, $\sigma$ significantly increases with increasing pressure along the whole $V_{G}$ range. $\mu$ estimated by Equation (2) shows a dependence on $V_{G}$. This dependence might originate from a considerable charge scattering at the interface to the Cytop insulator in the high $V_{G}$ region, which increases with increasing pressure. Accordingly, the mobility at $V_{G}=0 \mathrm{~V}$ increases by $30 \%$ (Figure 2a inset).

\section{Hall effect measurements}

Hall effect measurements are performed by varying temperature from room temperature down to $180 \mathrm{~K}$; the applied pressure is increased to $1 \mathrm{GPa}$. The magnetic field is swept back and forth at least three times in the range of -10 to $10 \mathrm{~T}$ to subtract the drifting signal for a precise evaluation of $\Delta V_{H}$ for the peak-to-peak magnetic field. The Hall coefficient $R_{H}$ is evaluated by $\Delta V_{H} /$ (I $\Delta B$ ), where $\Delta B$ is $20 \mathrm{~T}$. Typical data for the evolution of $V_{H}$ are shown in the Supplementary Materials (Supplementary Figure S2). As a result, $1 / R_{H}$ is obtained for each $V_{G}$, as shown in Figure $2 \mathrm{~b}$.

\section{Crystal structure analysis}

To assess the effect of thermal fluctuations, we analyze the temperature dependence of the fluctuations in pentacene on the basis of X-ray diffraction results performed at the SPring-8 facility (Supplementary Figure S3). The transfer integral is analyzed using density functional theory including van der Waals interaction, ${ }^{19,20}$ as described by Fukami et al. ${ }^{21}$ First, the fluctuation of the molecular motion is evaluated by defining all the degrees of freedom for the translational and librational motion; then, the fluctuation $(\Delta t)$ of the transfer integral $(t)$ is calculated because the transfer integral depends on the intermolecular distance and orientation. The accuracy of the calculated fluctuations of the molecular motion is confirmed by a translation-librationscrew analysis of the X-ray diffraction results. ${ }^{22}$

\section{RESULTS AND DISCUSSION}

\section{Emergence of fully coherent transport}

When comparing the measured curves, with the dashed line representing the induced charge density $Q$ in Figure $2 b$, a significant discrepancy from $1 / R_{H}$ is found. The situation obviously differs from the case of free-electron-like band transport, in which $1 / R_{H}$ equals $Q$. Given that the Hall voltage appears as a result of electromagnetic coupling based on coherent electronic waves, the numerical comparison between $1 / R_{H}$ and $Q$ gives a parameter to discuss the extent of the electronic coherence quantitatively. Here, we introduce the carrier 

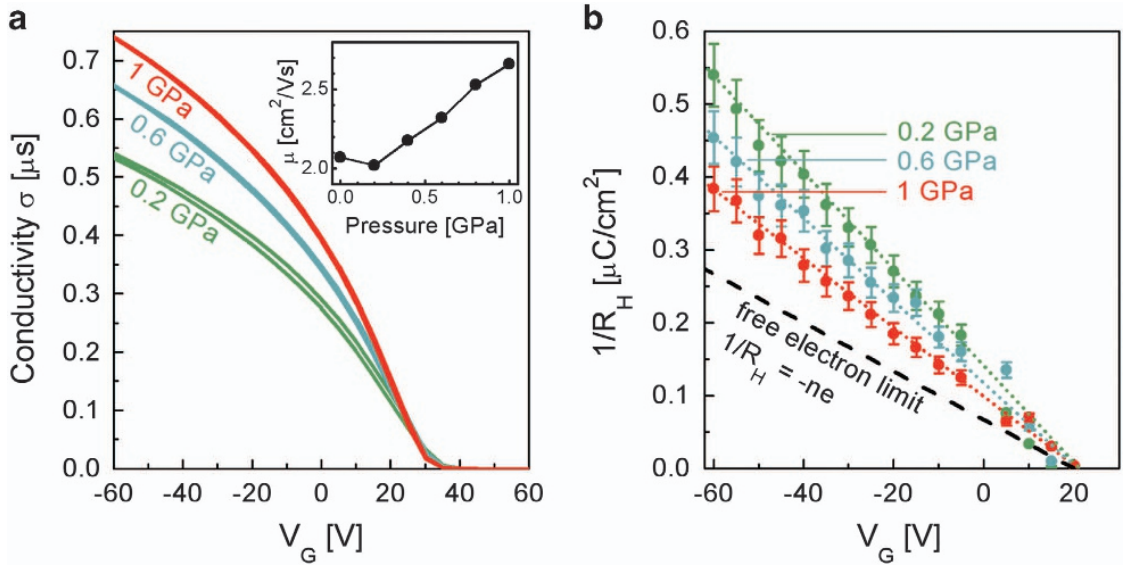

Figure 2 (a) FET transfer characteristics, i.e., conductivity $\sigma$ vs applied gate voltage $V_{G}$, measured using a four-terminal configuration to determine the carrier mobility. The inset shows an increase in the mobility by $30 \%$ upon application of pressure, exemplifying the effect of an increased molecular orbital overlap. (b) Inverse Hall coefficient as a function of the gate voltage $V_{G}$ at room temperature plotted together with the total induced charge carrier density (dashed line), which is the theoretical minimal value for $1 / R_{H}$ in the free electron limit. The higher the pressure, the closer the Hall coefficient approaches the free electron case. Obviously, increasing the pressure alone is not sufficient to induce fully coherent charge transport.
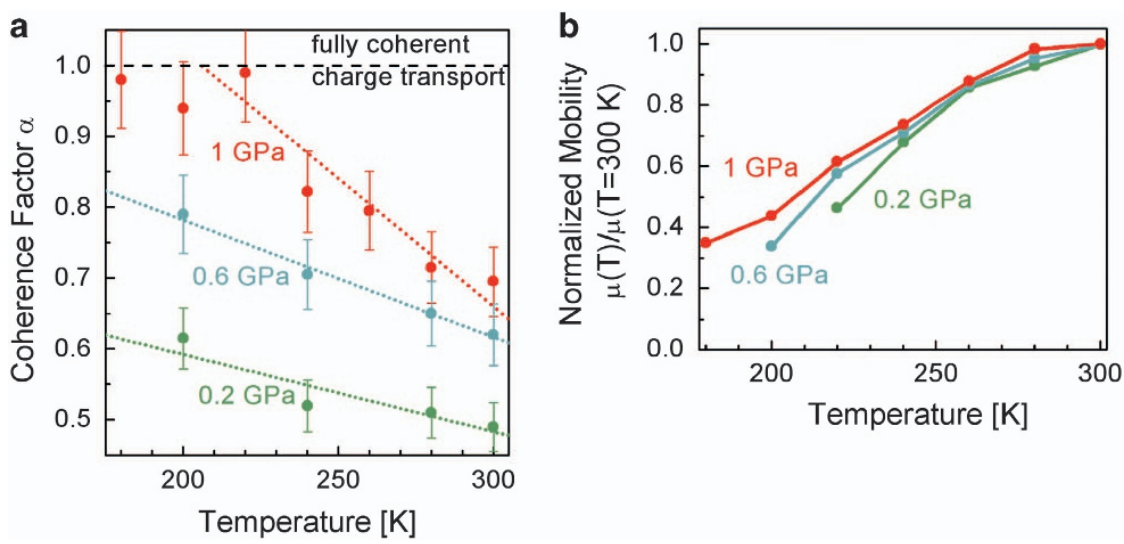

Figure 3 (a) Emergence of fully coherent charge transport in pentacene below $220 \mathrm{~K}$ at $1 \mathrm{GPa}$. By increasing the pressure and lowering the temperature, the thermal fluctuations are reduced, whereas, at the same time, the overlap of the molecular orbitals increases, improving charge coherence up to the point of full coherence $(\alpha=1)$. (b) Reduction of the temperature dependence of the normalized mobility $(\mu(\mathrm{T}) / \mu(\mathrm{T}=300 \mathrm{~K}))$ at higher pressure, indicative of an improved transfer integral between the pentacene molecules.

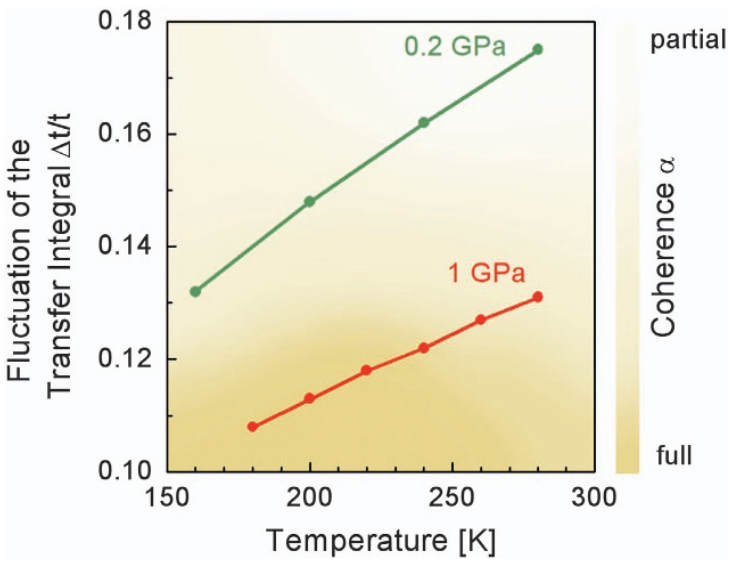

Figure 4 Reduction of the relative thermal fluctuation $\Delta t / t$ of the transfer integral at high pressure and low temperature. In the region of the emergence of coherent charge transport, the fluctuation is between 11 and $12 \%$, which appears to be the critical value for pentacene. coherent factor $\alpha$ as, ${ }^{5}$

$$
\alpha=\frac{C}{\delta\left(1 / R_{H}\right) / \delta V_{G}} .
$$

Equation (3) is the ratio of the slope between $1 / R_{H}$ as a function of $V_{G}$ and $Q=C V_{G}$. According to definition, $\alpha<1$ represents the situation where the electronic system cannot be described by fully coherent wavefunctions, while $\alpha=1$ represents systems with fully coherent charge transport as is observed in high-mobility organic semiconductors, such as rubrene, DNTT, alkyl-DNTT and pentacene derivatives. ${ }^{4-5,12-14}$ We note that the effect of inelastic scattering is minor in organic semiconductors, so that $\alpha>1$ is unlikely.

The coherence factor $\alpha$ gradually increases with increasing pressure (Figure 3a), indicating that the electronic charge coherence recovers when the molecular distance is reduced. The maximum value of $\alpha$ is still less than 1 at room temperature, that is, the electronic state is not yet completely the same, as is the case for rubrene single crystals. Interestingly, $\alpha$ increases gradually with increasing pressure and decreasing temperature, so that $\alpha$ reaches 1 below $220 \mathrm{~K}$ and at a 
pressure of $1 \mathrm{GPa}$. Thus, at high pressure and low temperature, pentacene single crystals exhibit a free electron-like band transport similar to rubrene, where full charge coherence already appears under ambient conditions. This observation suggests that the thermal fluctuations hinder coherent electronic states in pentacene.

The emergence of coherent electronic states directly reflects itself in an increased mobility. Using Equation (2), we calculate $\mu(\mathrm{P})$ at $V_{G}=0 \mathrm{~V}$ and plot the normalized temperature dependence under elevated pressure in Figure $3 \mathrm{~b}$. Although the overall temperature dependence of the normalized mobility $\mu$ is similar for all three curves, the measurement at $1 \mathrm{GPa}$ shows a weaker temperature dependence, indicative of a higher degree of coherence. We note that the mobility is a macroscopic property and is therefore affected by various extrinsic influences owing to inhomogeneities in the charge transport channel, such as micrometer-scale variation of the thickness in the polymer gate-insulating layer underneath the semiconductor. In contrast, the Hall effect measures nanoscale charge coherence over several molecules, thus revealing the intrinsic nature of the charge transport. In essence, these results suggest that, on a microscopic level, charge transport becomes fully coherent. On a macroscopic level, however, charge transport still seems to be dominated by incoherent hopping over static energy barriers. In absolute numbers, we see an increase in the mobility by $75 \%$ from 0.9 to $1.6 \mathrm{~cm}^{2} \mathrm{Vs}^{-1}$ at $220 \mathrm{~K}$ because of the increase of the coherence factor $\alpha$ from approximately 0.55 to 1 . At room temperature, we observe an increase in the mobility by $30 \%$ (Figure 2a), while the coherence factor $\alpha$ increases from 0.5 to 0.7 . Therefore, we can conclude that an increase of $\alpha$ by 0.1 leads to an increase in the mobility by approximately $15 \%$.

\section{Quantifying dynamic disorder}

It is known that pressure causes a reduction of the intermolecular distance by a few percent at $1 \mathrm{GPa},{ }^{23}$ increasing the intermolecular electronic coupling. This increased coupling is possible due to the van der Waals bonds resulting in a weak intermolecular repulsion. Indeed, the mobility significantly increases under pressure, demonstrating an enhanced transfer integral. As has been theoretically predicted, the effect of dynamic disorder as the result of molecular fluctuations is considerable at room temperature. ${ }^{2}$ By lowering the temperature, molecular fluctuations are suppressed, leading to an improved coherence which manifests itself in an enlarged $\alpha$. This temperature dependence is observed at all applied pressures until $\alpha$ saturates at a value of 1 at $1 \mathrm{GPa}$, indicating the emergence of fully coherent charge transport.

Because the value of $\Delta t / t$ is approximately $20 \%$ at room temperature, it can be presumed that the effect of thermal fluctuations is significant and therefore reduces the electronic coherence. Moreover, plotting the temperature dependence of $\Delta t / t$ in Figure 4, we notice that the value rapidly decreases towards $10 \%$, which is favorable for the emergence of coherent electronic states. These observations show that reducing the dynamic disorder resulting from thermal fluctuations is important to observe coherent charge transport. Thus, synthesizing molecules with steric hindrance is crucial to achieving full coherence under ambient conditions, an ongoing topic in research. ${ }^{13,24-27}$

\section{CONCLUSION}

The observations in this experiment are a clear manifestation of the charge transport mechanism in soft organic semiconductors. A slightly modified packing structure allows for a continuous evolution from an incomplete coherence up to band transport. This transition was achieved by increasing the pressure and lowering the temperature, thus reducing the intermolecular distance and improving the electronic coupling, manifesting itself as enhanced mobility. In addition, the vibrational modes of the molecules that lead to dynamic disorder are suppressed, thus improving the coherence of electronic states. This emergence of charge coherence is accompanied by a mobility increase of $75 \%$. In a detailed analysis, we quantified the effect of thermal fluctuations on charge transport, revealing that the critical value for the variation of the transfer integral at which coherence emerges is below $12 \%$ of its mean value. These surprising findings show that pentacene is the missing link between coherent and incoherent charge transport, a crucial foundation for further studies of the underlying charge transport mechanisms in 'soft' semiconductors and an important aspect for further improvement of their performance.

\section{CONFLICT OF INTEREST}

The authors declare no conflicts of interest.

\section{ACKNOWLEDGEMENTS}

This work was financially supported by a Grant-in-Aid for Scientific Research (No. 26246011) from MEXT, Japan. RH acknowledges the financial support by the Japan Society for the Promotion of Science under their International Research Fellow program.

Author contributions: KS conceived of, planned and executed the experiments, analyzed and interpreted the results and wrote the manuscript. YO, TU, JT, HM, TF, HI, NK, KH and JT provided support and technical discussions. RH edited the manuscript.

1 Pope, M. \& Swenberg, C. E. Electronic Processes in Organic Crystals and Polymers (Oxford Univ. Press, Oxford, UK, 1999).

2 Troisi, A. \& Orlandi, G. Charge-transport regime of crystalline organic semiconductors: diffusion limited by thermal off-diagonal electronic disorder. Phys. Rev. Lett. 96, 086601 (2006).

3 Takeya, J., Tsukagoshi, K., Aoyagi, Y., Takenobu, T. \& Iwasa, Y. Hall effect of quasihole gas in organic single-crystal transistors. Jpn. J. Appl. Phys. 44, L1393-L1396 (2005).

4 Podzorov, V., Menard, E., Rogers, J. \& Gershenson, M. Hall effect in the accumulation layers on the surface of organic semiconductors. Phys. Rev. Lett. 95, 226601 (2005).

5 Takeya, J., Kato, J., Hara, K., Yamagishi, M., Hirahara, R., Yamada, K., Nakazawa, Y., Ikehata, S., Tsukagoshi, K., Aoyagi, Y., Takenobu, T. \& Iwasa, Y. In-crystal and surface charge transport of electric-field-induced carriers in organic single-crystal semiconductors. Phys. Rev. Lett. 98, 196804 (2007).

6 Friedman, L. Hall conductivity of amorphous semiconductors in the random phase model. J. Non. Cryst. Solids 6, 329-341 (1971).

7 Wang, S., Ha, M., Manno, M., Frisbie, D. \& Leighton, C. Hopping transport and the Hall effect near the insulator-metal transition in electrochemically gated poly(3-hexylthiophene) transistors. Nat. Commun. 3, 1210 (2012).

8 Yamashita, Y., Tsurumi, J., Hinkel, F., Okada, Y., Soeda, J., Zajączkowski, W., Baumgarten, M., Pisula, W., Matsui, H., Müllen, K. \& Takeya, J. Transition between band and hopping transport in polymer field-effect transistors. Adv. Mater. 26, 8169-8173

(2014)

9 Menard, E., Podzorov, V., Hur, S.-H., Gaur, A., Gershenson, M. E. \& Rogers, J. A. High-performance $\mathrm{n}$ - and $\mathrm{p}$-Type single-crystal organic transistors with free-space gate dielectrics. Adv. Mater. 16, 2097-2101 (2004)

10 Takeya, J., Yamagishi, M., Tominari, Y., Hirahara, R., Nakazawa, Y., Nishikawa, T., Kawase, T., Shimoda, T. \& Ogawa, S. Very high mobility organic single crystal transistors with in-crystal conduction channels. Appl. Phys. Lett. 90, 102120 (2007).

11 Reese, C., Chung, W.-J., Ling, M. M., Roberts, M. \& Bao, Z. High-performance microscale single-crystal transistors by lithography on an elastomer dielectric. Appl. Phys. Lett. 89, 202108 (2006).

12 Yamagishi, M., Soeda, J., Uemura, T., Okada, Y., Takatsuki, Y., Nishikawa, T., Nakazawa, Y., Doi, I., Takimiya, K. \& Takeya, J. Free-electron-like Hall effect in high-mobility organic thin-film transistors. Phys. Rev. B 81, 161306 (2010).

13 Mitsui, C., Okamoto, T., Yamagishi, M., Tsurumi, J., Yoshimoto, K., Nakahara, K., Soeda, J., Hirose, Y., Sato, H., Yamano, A., Uemura, T. \& Takeya, J. High-performance solution-processable n-shaped organic semiconducting materials with stabilized crystal phase. Adv. Mater. 26, 4546-4551 (2014).

14 Chang, J.-F., Sakanoue, T., Olivier, Y., Uemura, T., Dufourg-Madec, M.-B., Yeates, S. G., Cornil, J., Takeya, J., Troisi, A. \& Sirringhaus, H. Hall-effect 
measurements probing the degree of charge-carrier delocalization in solution-processed crystalline molecular semiconductors. Phys. Rev. Lett. 107, 066601 (2011).

15 Minder, N. A., Ono, S., Chen, Z., Facchetti, A. \& Morpurgo, A. F. Band-like electron transport in organic transistors and implication of the molecular structure for performance optimization. Adv. Mater. 24, 503-508 (2012).

16 Uemura, T., Yamagishi, M., Soeda, J., Takatsuki, Y., Okada, Y., Nakazawa, Y. \& Takeya, J. Temperature dependence of the Hall effect in pentacene field-effect transistors: possibility of charge decoherence induced by molecular fluctuations. Phys. Rev. B 85, 035313 (2012).

17 Okada, Y., Sakai, K., Uemura, T., Nakazawa, Y. \& Takeya, J. Charge transport and Hal effect in rubrene single-crystal transistors under high pressure. Phys. Rev. B 84, 245308 (2011).

18 Sakai, K., Okada, Y., Kitaoka, S., Tsurumi, J., Ohishi, Y., Fujiwara, A., Takimiya, K. \& Takeya, J. Anomalous pressure effect in heteroacene organic field-effect transistors. Phys. Rev. Lett. 110, 096603 (2013).

19 Schmidt, M. W., Baldridge, K. K., Boatz, J. A., Elbert, S. T., Gordon, M. S. Jensen, J. H., Koseki, S., Matsunaga, N., Nguyen, K. A., Su, S. J., Windus, T. L., Dupuis, M. \& Montgomery, J. A. General atomic and molecular electronic structure system. J. Comput. Chem. 14, 1347 (1993).

20 Grimme, S. Accurate description of van der Waals complexes by density functional theory including empirical corrections. J. Comput. Chem. 25, 1463 (2004).

21 Fukami, T., Ishii, H., Kobayashi, N., Uemura, T., Sakai, K., Okada, Y., Takeya, J. \& Hirose, K. Correlation between thermal fluctuation effects and phase coherence factor in carrier transport of single-crystal organic semiconductors. Appl. Phys. Lett. 106, 143302 (2015).

22 Schomaker, V. \& Trueblood, K. N. On the rigid-body motion of molecules in crystals. Acta Cryst B24, 63-76 (1968).
23 Oehzelt, M., Aichholzer, A., Resel, R., Heimel, G., Venuti, E. \& Della Valle, R. G. Crystal structure of oligoacenes under high pressure. Phys. Rev. B 74, 104103 (2006).

24 Sakanoue, T. \& Sirringhaus, H. Band-like temperature dependence of mobility in a solution-processed organic semiconductor. Nat. Mater. 9, 736-740 (2010).

25 Minder, N. A., Lu, S., Fratini, S., Ciuchi, S., Facchetti, A. \& Morpurgo, A. F. Tailoring the molecular structure to suppress extrinsic disorder in organic transistors. Adv. Mater. 26, 1254-1260 (2014).

26 Okamoto, T., Mitsui, C., Yamagishi, M., Nakahara, K., Soeda, J., Hirose, Y., Hirose, Y. Miwa, K., Sato, H., Yamano, A., Matsushita, T., Uemura, T. \& Takeya, J. V-shaped organic semiconductors with solution processability, high mobility, and high thermal durability. Adv. Mater. 25, 6392-6397 (2013).

27 Uemura, T., Nakayama, K., Hirose, Y., Soeda, J., Uno, M., Li, W., Yamagishi, M. Okada, Y. \& Takeya, J. Band-like transport in solution-crystallized organic transistors. Curr. Appl. Phys. 12, 87-91 (2012).

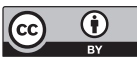

This work is licensed under a Creative Commons Attribution 4.0 International License. The images or other third party material in this article are included in the article's Creative Commons license, unless indicated otherwise in the credit line; if the material is not included under the Creative Commons license, users will need to obtain permission from the license holder to reproduce the material. To view a copy of this license, visit http:// creativecommons.org/licenses/by/4.0/

Supplementary Information accompanies the paper on the NPG Asia Materials website (http://www.nature.com/am) 